\title{
NORMATIVA PARA LA PUBLICACIÓN DE ARTÍCULOS EN LA REVISTA ALCANCES TECNOLÓGICOS
}

\section{ASPECTOS GENERALES}

1. La edición de la revista es una de las actividades relevantes del área de transferencia de tecnología del INTA-Costa Rica, por lo que se publicará un número por año.

2. Únicamente se aceptarán aquellos artículos que no hayan sido publicados en otra(s) revista(s).

3. La revista tiene carácter técnico-científico y en ella se pueden publicar:

\section{- Artículos formales}

Se refiere a una investigación profunda y detallada con todos los elementos de un artículo científico (resumen, introducción, materiales y métodos, resultados y discusión y literatura citada). Se debe demostrar la profundidad del estudio y resaltar los méritos del trabajo para su publicación en una revista científica.

\section{- Comunicaciones cortas}

Son resultados preliminares de urgencia e interés. No debe ser estructurada de la misma manera que un artículo formal, debe contener introducción y resultados.

\section{- Notas técnicas}

Se refiere a la publicación de técnicas o metodologías innovadoras. La extensión máxima es de cinco páginas.

\section{- Revisiones bibliográficas}

Son recopilaciones y síntesis del conocimiento existente en un campo específico de interés en las ciencias agrícolas.

\section{-Análisis y comentarios}

Es el análisis de una situación específica, realizado por un especialista con reconocida trayectoria en el campo.

\section{- Informaciones técnicas}

Están enfocadas en aprovechar la amplia experiencia de un experto en un campo específico (Araya 2009).

\section{PROCEDIMIENTOS}

1. La aceptación o no de los escritos será de acuerdo con las normas y procedimientos para publicar artículos científicos y es competencia del Comité Editorial del INTA.

2. Una vez que el Comité Editorial recibe el artículo, dispone de un mes para enviarlo a los revisores. Los revisores internos tendrán un plazo máximo de un mes para entregar las publicaciones revisadas con un informe escrito de las mismas. A los revisores externos se les sugerirá el mismo tiempo para revisarlo.

3. Cuando el artículo es devuelto por los revisores, la editora o el editor dispondrá de ocho días hábiles para enviarlo a los autores con una nota en la que se indican las correcciones respectivas. Por su parte, los autores contarán con un plazo máximo de 15 días hábiles para hacer las correcciones y devolverlo, a la editora o al editor.

4. Los artículos científicos deben tener una extensión máxima de 20 páginas escritas a doble espacio, en Microsoft Word con letra Arial 12.

5. Las notas técnicas deben tener una extensión no mayor de 12 páginas escritas a doble espacio, con el mismo tipo de letra. 
6. En la redacción de los artículos se deben utilizar las normas de la Real Academia Española y las unidades de medida del Sistema Métrico Decimal.

- Las unidades no llevan punto, se escriben con minúscula y no tienen plural. Algunos ejemplos son: kilogramo $(\mathrm{kg})$, gramo $(\mathrm{g})$, metro $(m)$, hectárea (ha), grados Celsius (C), milímetro ( $\mathrm{mm}$ ), miligramo $(\mathrm{mg})$ litro (I), metros sobre el nivel del mar (msnm), elementos ( $N, P$ entre otros), compuestos químicos (como por ejemplo: $\mathrm{NaOH}, \mathrm{NaCl}$ ).

- Cuando las unidades no están precedidas por un número, se expresan por su nombre completo sin utilizar su abreviatura. Por ejemplo: metro en lugar de $\mathrm{m}$.

- Los decimales se indican con coma; los miles y los millones con un espacio. Ejemplo: 8327 451,25. Los números de cuatro cifras se escriben sin espacios. Ejemplo: 2458.

- En el caso de los números del cero al nueve, cuando no van seguidos de unidades, se escriben con palabra; y números para valores iguales o mayores a diez.

7. Cuando se citan plaguicidas se debe utilizar solo los nombres genéricos del producto. Ejemplos: Terbufos, Oxidemeton Metil. No se acepta el uso de nombres comerciales excepto en el caso que sean formulaciones particulares que influyen en los resultados.

\section{ESTRUCTURA DE LOS ARTÍCULOS}

\section{Título}

Tiene que ser breve, específico y resumido. No más de 14 palabras. Indicar los nombres científicos en cursiva y negrita (cuando el nombre común no es muy conocido), en el texto solamente en cursiva. Las palabras del título no se repiten en las palabras clave.
- Estudio sobre.......

- Informe de....

- Investigación acerca de

- Contribución a.

- Resultados de un estudio sobre.......

-Análisis de los resultados.........

Los nombres científicos (género, especie, cultivar y el nombre del clasificador) deberán ser citados para cada organismo en su primera mención, posteriormente se puede continuar usando el nombre común. Se escriben con letra cursiva.

\section{Autor(es)}

Se considera ( $n$ ) autor (es), el (los) individuo (s) (autor (es) personal (es), o la entidad (es), institución (es), asociación(es), organización (es), sociedad (es) (autor (es) corporativo (es), responsable (s) de los contenidos intelectuales de las publicaciones.

El orden en el que se mencionan va de acuerdo con su contribución y aportes en la investigación y se colocan debajo del título. Con una nota al pie de página indicando la institución para la cual labora el (los) autor (es). Se omiten los grados académicos del (los) autor (es). Si el (los) autor (es) lo desea $(n)$ puede $(n)$ indicar la dirección electrónica.

\section{Resumen:}

Se coloca después del nombre de los autores y presenta en forma concisa el mensaje del artículo, describiendo brevemente los materiales y condiciones más relevantes del experimento. Debe indicar el año y lugar, los resultados obtenidos y las conclusiones más importantes. Las oraciones usadas deben ser racionales, objetivas y justificar el porqué de la investigación y el objetivo, evitando describir directamente los materiales y métodos. La extensión no debe exceder las 250 palabras. Debajo del resumen se colocan las palabras clave.

Lo que no se usa en los títulos (Araya 2012)․․

\footnotetext{
${ }^{1}$ Araya R. 2012. Lo que no se usa en los títulos. (entrevista). San José, CR. Comunicación Personal.
} 


\section{Introducción}

Define el problema que motiva la investigación y al final de esta sección se indican los objetivos o razones del estudio. Pueden incluirse citas bibliográficas para ayudar a la definición del problema y del trabajo. La extensión de ésta se recomienda sea de aproximadamente 350 palabras (MAG 1990).

\section{Materiales y Métodos}

Describen en forma bien detallada la ubicación, la fecha de inicio y término, el ambiente, los materiales, las técnicas, los tratamientos, el diseño experimental, los análisis estadísticos y las variables a evaluar expuestas con suficiente claridad para que otros científicos puedan repetir el estudio. Si el método es muy conocido, solamente se incluyen referencias bibliográficas aclaratorias; si es nuevo o modificado se debe escribir nuevamente. Escribir en orden cronológico (MAG 1990).

\section{Resultados y Discusión}

Se recomienda que ambas partes vayan juntas. Los resultados describen la información generada por la investigación; debe escribirse en forma concisa y siguiendo una secuencia lógica, usando cuadros y figuras (cuando se incluyen fotografías, se les da el nombre de figuras y su numeración se debe ajustar a la secuencia de los gráficos). Los cuadros se presentan sin divisiones internas. Los cuadros y figuras deben estar ubicados donde se mencionan, deben ser auto explicativos y la información debe presentarse en forma completa, clara y concisa, de tal forma que no se tenga que recurrir al texto para entender el resultado presentado. Use decimales cuando sea justificado, si no, redondee o aproxime apropiadamente. Además de la descripción del contenido de la figura, en el título debe contener el lugar y el año en que se hizo el trabajo de investigación.
En la discusión no abuse de la estadística, úsela como una herramienta para probar la(s) hipótesis propuesta(s), con una base objetiva. Suministrar la significancia de las pruebas.

Se discutirán los resultados obtenidos, comparándolos con otros trabajos afines para dar interpretaciones 0 hacer deducciones lógicas sobre las diferencias o concordancias encontradas.

En la "Discusión" se debe explicar hasta qué punto los resultados obtenidos contribuyen a la solución del problema (limitantes) y qué puede traducirse en recomendaciones, aplicaciones, sugerencias e hipótesis (MAG 1990).

\section{Conclusiones}

Van incluidas en la discusión

\section{REFERENCIAS BIBLIOGRÁFICAS (LITERATURA CITADA)}

La lista de la literatura citada debe estar conformada por no menos de diez citas bibliográficas recientes y se deben utilizar las NORMAS DE REDACCIÓN (IICA-CATIE) en su 4 ed.

\section{- Libros y Folletos}

La portada es la fuente principal de la información para redactar la referencia, sin embargo, hay otras partes como la cubierta, la falsa portada, el colofón, la solapa, la introducción, etc.

Los elementos son:

Autor(es). Año de publicación. Título: Subtítulo. Mención del traductor y /o editor. Edición. Ciudad y/o país de publicación en caso necesario, Casa editora. Páginas o volúmenes (Mención de serie).

Crosby, PB.1990. Dinámica gerencial: el arte de hacer que las cosas ocurran. México, DF, Mc Graw-Hill.272p. (Serie de Administración). 


\section{- Tesis}

Se elabora de la misma forma que la de los libros y folletos, pero después del título se anota la palabra Tesis seguida del grado académico en forma abreviada, en el idioma en que está escrita la tesis.

Autor(es). Año de publicación. Título: subtítulo. Mención del grado académico. Ciudad y país donde se ubica la institución, Nombre de la institución que otorga el grado. Páginas.

Yah Correa, E. V.1988. Crioconservación de suspensiones celulares embriogénicas de Musa spp iniciadas a partir de flores inmaduras. Tesis Mag. Sc. Turrialba, CR, CATIE. 77 p.

\section{- Conferencias, Congresos, Reuniones y otros}

Los informes, memorias, actas, resúmenes de las conferencias, congresos, reuniones, simposios, nacionales e internacionales se anotan por el mismo nombre de la conferencia, congreso, o reunión.

\section{Los elementos son:}

Nombre del evento (Número, Año de realización, Lugar donde se realizó). Año de publicación. Título. Mención del Editor (es). Ciudad y país de publicación, Casa editorial. Páginas o volúmenes.

Regional Workshop Needs and Priorities for Forestry and Agroforestry Policy Research in Latin América (1993 San José, CR). 1994. (Report). Eds. M Alfaro, R de Camino, M. I. Mora, P Oram. San José, CR, IICA. 298 p.

\section{- Analíticas}

\section{Obra colectiva}

Es la referencia biográfica de un trabajo escrito por un autor en un documento editado o compilado por otro(s) autor(es) tal y como es el caso de las conferencias, reuniones o congresos.

Los elementos son:

Autor, Año de publicación .Título del trabajo consultado. Preposición latina In, la referencia bibliográfica completa de la fuente que lo contiene, con las páginas iniciales y finales de la parte analizada sin mencionar nuevamente el año de publicación.

Mortimer, A.M.1990.Thebiologyofweeds. In Hance, JR; Holly, K. eds. Weed control handbook: principals. 8 ed. Oxford, GB, British Crop Protection Council. p. 1-42.

Santos Pereira, H dos. 1997. Brasil. In Reunión de los puntos focales de los Programas forestales nacionales de América Latina y el Caribe (1997, Brasilia, DF). Memoria. Santiago, CL. p. 49-56.

\section{Trabajo de un autor en su propia obra.}

La redacción de la referencia bibliográfica de una parte o capítulo con título específico escrito por un autor en una obra propia, tiene los elementos siguientes:

Autor. Año de publicación. Título de la parte o capítulo. Preposición In y los datos que incluye la referencia bibliográfica completa del libro o folleto sin mencionar nuevamente el autor ni el año de publicación. El autor se vuelve a mencionar en el caso que la publicación contenga más de un autor o un editor.

Phetig, R. 1994. Valuing the environmental methodological and measurement issues. In Ecological dynamics and the valuation of environmental change. Dordrecht, kluwer. $p$. 3-22.

Mugabe, J.; Otieno-Odek, J. 1997. National access regimes: capacity building and policy reforms. In: Mugabe, J; Barber, CV; Henne, G; Glowka, L. eds. Access to genetic resources. Nairobi, ACTC. p. 95-41. 


\section{- Publicación periódica}

Es aquella obra editada por lo general con título distintivo, en fascículos o partes a intervalos regulares, en orden numérico - cronológico y que pretende continuar indefinidamente. Incluye trabajos sobre temas diversos en un solo ejemplar, con la colaboración de varios autores (revistas, periódicos diarios).

\section{Revistas}

Elementos:

Autor(es). Año de publicación. Título del artículo. Nombre de la revista Volumen de la revista (Número de la revista): página inicial y final del artículo.

El volumen y el número se mencionan en números arábigos.

Singh, CK.; Grewal, GS. 1998. Detection of rabies in central nervous system of experimentally infected buffalo calves. Indian Journal of Animal Sciences 68(12):1242-1254.

\section{Sin Volumen y sin número}

Se recurre a algún elemento que pueda ayudar a su identificación, como son los meses o las estaciones del año.

Powles, H. 1987. Fencing off fish. Caribbean Farming feb. 1987. 13, 21.

Con Volumen sin número

$\mathrm{Si}$ la revista tiene solamente el volumen se indica dicho dato, sin ninguna abreviatura.

Pierce, F. 1999. Aspects of precision agriculture. Advances in Agronomy 67:1-58

Sin Volumen con número

Se utiliza la abreviatura "no" antes de dicho número.

Chamorro-Trejos, G. 1993. Zoca de café intercalada con nogal. Bosques y Desarrollo no. 9:46-49

\section{Periódicos o diarios}

Elementos:

Autor(es). Año de publicación del periódico. Título del artículo. Nombre del periódico, Ciudad de publicación, país abreviado, mes abreviado. Día: página.

Méndez, W. 1998. Prometen apoyo a cooperativismo. La Nación, San José, CR, ene.8:6A.

\section{Separatas}

La cita se hace según las normas establecidas para cada tipo de material. La fuente donde fue originalmente publicado el trabajo debe indicarse en una nota y en el idioma en que se redacta la bibliografía.

Sánchez, P. 1995. Science in Agroforestry. Nairobi, ICRAF. 50 p. Reimpreso de: Agroforestry Systems 30:5-55.

\section{Materiales cartográficos}

Incluyen mapas o atlas de países, regiones, áreas y continentes; mapas o atlas básicos con datos estadísticos; estudios de observación en agricultura; cartas meteorológicas o hidrográficas, fotografías aéreas con fines cartográficos y otros.

Elementos:

Autor(es). Año de publicación. Título. Edición. Lugar de publicación, Casa editorial. Escala. Paginación. Indicación de color (Serie).

Cortés, G. 1994. Atlas agropecuario de Costa Rica. San José, CR, EUNED. Ese. varía. 513 p. Color COSEFORMA (Cooperación en los Sectores Forestal y Maderero, CR). Convenio Costarricense Alemán. 1996. Zonas bioclimáticas de la región Huetar Norte de Costa Rica. San José, CR. Esc. 1:200.000. Color. 


\section{Material Audiovisual}

Materiales gráficos $\begin{array}{r}\text { (fotobandas, } \\ \text { diapositivas, transparencias, fotografías, }\end{array}$
diagramas y otros) y colecciones de estos
materiales; grabaciones sonoras (cintas,
cáseles, discos), microfichas, micropelículas,
películas y videograbaciones.

Elementos:

Autor(es). Año de publicación. Título: subtítulo. Mención del traductor y/ o editor. Edición. Ciudad y país de publicación, Casa editora. Descripción física (Mención de serie).

\section{Microficha}

FAO (Organización de las Naciones Unidas para la Agricultura y la Alimentación, IT).1990. Guidelines for soil profile description (microficha). 2 ed. Roma. 10,5 x 14,5 cm.

\section{Diapositiva}

CATIE (Centro Agronómico Tropical de Investigación y Enseñanza, CR). 1990. La investigación silvicultural (diapositivas). Turrialba, CR.110 diapositivas, son. 1 casete (26 min.), color.

\section{Videocinta}

Instituto para el Desarrollo de Sistemas de Producción del Trópico Húmedo de Tabasco, MX.1995. La mujer y la Agricultura. Tabasco, MX. (videocasete). 1 videocinta VHS (10:49 $\min$ ), son., color.

\section{Documentos Electrónicos}

Actualmente en forma electrónica se encuentran monografías, publicaciones periódicas, mensajes, conferencias, reuniones, bases de datos, programas de computadora, etc. Por tanto, se seguirán las normas establecidas para cada uno de ellos y además se incluirán otros elementos que permitan identificar el medio en que están disponibles (en línea, disco compacto, disquetes, mensajes electrónicos, cintas magnéticas y otros).

\section{Elementos:}

Autor(es). Año de publicación. Título: subtítulo. (Tipo de medio). Edición. Ciudad y país de publicación, Casa editora. Fecha en que se consultó el material para los documentos en línea. Descripción física. Disponibilidad y acceso para los documentos en línea. (Nota de serie).

\section{En línea}

Documento disponible en línea a través de los servicios de internet.

\section{Libros}

Guzmán, M de. 1993. Tendencias innovadoras en educación matemática, (en línea).Bogotá, Unesco. Consultado 5 ene. 1998. Disponible en http://www.oel.org.co/ oeivirt/edumat.htm.

\section{Revistas}

Rodríguez, I. 1999. Tratamientos del agua potable, (en línea). Globo Terráqueo No. 20610. Consultado 10 set. 1999. Disponible en http://www.interbook.net/personal/ jigonzales1set99.htp

\section{Base de datos}

Fundación Arias para la paz y el progreso humano, CR. 1998. Ceiba: base de datos.

ONG centroamericanas, (en línea). San José, CR. Consultado 15 ene. 1998. Disponible en http://www.arias.or.cr./ceiba.

\section{Correo electrónico}

Núñez, R. 1999. Plan de trabajo SIDALC. (correo electrónico). Santo Domingo, RD, IICA.

\section{Disco compacto}

Frater, H; Paulissen, D. 1995. El gran libro de multimedia. México, DF. Computec. 1 disco compacto, $8 \mathrm{~mm}$.

\section{Comunicaciones Personales}

No deben figurar en la literatura citada, se mencionan en nota al pie de página en el texto de la publicación. 
Elementos:

Autor. Año en que tuvo lugar la comunicación. Título de la comunicación. Lugar, e institución donde trabaja el autor. Mención de Comunicación personal.

Aguilar, JF. 1997. Forestería social (entrevista). San José, CR, Universidad de Costa Rica. Comunicación Personal.

Salazar, F. 1999. Formación de consorcios (correo electrónico). Bogotá. Comunicación Personal.

\section{Notas}

Son datos suplementarios sobre el contenido o ciertas características especiales de un documento, que se agregan a la referencia para aclarar y ampliar información cuando es necesario.

Las hay de dos tipos: Notas de contenido y Notas sobre las características específicas de la publicación.

- Notas de contenido

- Notas sobre las características específicas de la publicación.

Trabajos sin publicar

Si un trabajo no se ha publicado o está en proceso de publicación, se agrega la frase: en prensa o sin publicar.

Somarribas, E. 1997. Shade management in coffee and cocoa plantations. Agroforestry Systems. En prensa.

\section{Presentación, ordenación y organización de la lista bibliográfica}

Se presenta al final del trabajo y se le asigna el título de: Literatura Citada.

Hay diversas formas de organizaría según el uso que se le vaya a dar; sin embargo en los trabajos científicos y técnicos predomina el arreglo alfabético por autor y en orden cronológico por año de publicación iniciando con la más antigua para finalizar con la más reciente.

\section{Citas de un mismo autor publicadas el mismo año}

Luna, A. 1995a. El bosque protector. Mérida, VE, Instituto Forestal Latinoamericano. $71 \mathrm{p}$.

Luna, A. 1995b. Ordenación sostenible de los bosques naturales en Venezuela. Criterio para la evaluación de la ordenación sostenible de los bosques tropicales: caso de Venezuela. Mérida, VE. Instituto Forestal Latinoamericano. 68 p.

Si alguna de las citas de un mismo autor no tiene fecha de publicación, se coloca primero que las demás.

Formas de citar las referencias bibliográficas dentro del texto.

Por cuestiones de ética y derechos de autor todo investigador debe dar crédito de los trabajos que ha utilizado para desarrollar su investigación, facilitando con ello identificar a los autores de planteamientos y resultados anteriores que fundamentan dicha investigación.

Hay diferentes modos de citación en el texto que varían según las disciplinas. No obstante, en el caso de trabajos científicos y técnicos el que más se emplea es el Sistema autorfecha. Consiste en referenciar un trabajo, del texto a la lista bibliográfica publicada al final de la publicación, por medio del apellido (s) del autor (es) seguido por el año de publicación.

\section{Cita contextual}

En la redacción de cualquier trabajo de investigación se emplea con mucha frecuencia la cita contextual. La cita contextual es aquella en que un autor toma una idea, un resultado o un punto de vista de otro autor y lo presenta en sus propias palabras para reforzar o aclarar su 
propia investigación. Puede redactarse de dos maneras:

Haciendo énfasis en el autor

Es cuando el nombre del autor va incluido en la redacción del párrafo.

Brenes (1998) ha demostrado que las variedades de mayor rendimiento son más susceptibles al ataque de nematodos.

Estudios realizados por Brenes (1998) muestran que las variedades de mayor rendimiento son más susceptibles al ataque de nematodos.

Haciendo énfasis en el texto

Es cuando se redacta el párrafo sin mencionar el autor. Este se indica entre paréntesis al final del párrafo.

Las variedades de mayor rendimiento son más susceptibles al ataque de nematodos (Brenes 1998).

Ejemplos con variaciones

Publicación con un autor

Finegan (1992) demostró que el rendimiento...

El mejoramiento genético da mejor rendimiento... (Finegan 1992) Estudios realizados por Rivas Platero (1995) sobre micorrizas... Avances de investigación en micorrizas... (Rivas Platero 1995)

\section{Publicación con dos autores}

En el caso de dos autores de una misma publicación se cita por los apellidos de ambos unidos por la conjunción "y".

Rodríguez y Salas (1993) determinaron que la rentabilidad de los sistemas agroforestales.

Considerando la rentabilidad de los sistemas agroforestales... . (Rodríguez y Salas 1993)

\section{Publicación con tres o más autores}

En el caso de tres o más autores de una misma publicación se cita por el apellido(s) del primer autor seguido por la expresión latina et al. (y otros).

Estudios realizados por Salazar et al. (1994) sobre la densidad de adultos virulíferos... .

La densidad de adultos virulíferos de Bemisia... . (Salazar et al. 1994).

Más de una cita o publicación

Cuando se requiere citar más de una publicación a la vez, se debe separar cada una de ellas por coma (,). Las publicaciones deben mencionarse en orden cronológico por fecha de publicación, de la cita más vieja a la más reciente.

Ruíz (1980), García y Sánchez (1992) y Rojas (1996) analizaron muestras de suelos...

Fertilización con N, P, K aplicadas a muestras de suelos. (Ruíz 1980, García y Sánchez 1992, Rojas 1996)

\section{LITERATURA CITADA}

IICA (Instituto Interamericano de Cooperación para la Agricultura, CR); CATIE (Centro Agronómico Tropical de Investigación y Enseñanza, CR). 1999. Redacción de referencias bibliográficas: normas técnicas del IICA y CATIE. 4 ed. Costa Rica Biblioteca Conmemorativa Orton. p. 25.

MAG (Ministerio de Agricultura y Ganadería). 1990. Normas para la publicación de artículos científicos en la revista investigación agrícola. Investigación Agrícola 4(2):3-6.

Araya, R. 2009. Instructivo para los autores. Agronomía Mesoamericana. 20(2):433-436. 